\title{
Heliconius butterflies host characteristic and phylogenetically structured adult-stage microbiomes
}

Tobin J. Hammer ${ }^{*}$, Jacob C. Dickerson ${ }^{2,3}$, W. Owen McMillan², Noah Fierer ${ }^{3,4}$

1. Department of Integrative Biology, University of Texas at Austin, Austin, TX 78712, USA

2. Smithsonian Tropical Research Institute, Apartado 0843-03092, Panama City, Panama

3. Department of Ecology \& Evolutionary Biology, University of Colorado at Boulder, Boulder, CO 80309, USA

4. Cooperative Institute for Research in Environmental Sciences, University of Colorado at Boulder, Boulder, CO 80309, USA

*Corresponding author: tobin.hammer@utexas.edu

Keywords: symbiosis, gut bacteria, Lepidoptera, microbiota, parasites 


\section{Abstract}

Lepidoptera (butterflies and moths) are diverse and ecologically important, yet we know

4 little about how they interact with microbes as adults. Due to metamorphosis, the form and

5 function of their adult-stage microbiomes might be very different from microbiomes in the larval

6 stage (caterpillars). We studied adult-stage microbiomes of Heliconius and closely related

7 passion-vine butterflies (Heliconiini), which are an important model system in evolutionary

8 biology. To characterize the structure and dynamics of heliconiine microbiomes, we used field

9 collections of wild butterflies, 16S rRNA gene sequencing, quantitative PCR, and shotgun

10 metagenomics. We found that Heliconius harbor simple and abundant bacterial communities that

11 are moderately consistent among conspecific individuals and over time. Heliconiine

12 microbiomes also exhibited a strong signal of host phylogeny, with a major distinction between

13 Heliconius and other butterflies. These patterns were largely driven by differing relative

14 abundances of bacterial phylotypes shared among host species and genera, as opposed to the

15 presence or absence of host-specific phylotypes. We suggest that phylogenetic structure in

16 heliconiine microbiomes arises from conserved host traits that differentially filter microbes from

17 the environment. While the relative importance of different traits remains unclear, our data

18 indicate that pollen-feeding (unique to Heliconius) is not a primary driver. Using shotgun

19 metagenomics, we also discovered trypanosomatids and microsporidia to be prevalent in

20 butterfly guts, raising the possibility of antagonistic interactions between eukaryotic parasites

21 and co-localized gut bacteria. Our discovery of characteristic and phylogenetically structured

22 microbiomes provides a foundation for tests of adult-stage microbiome function, a poorly

23 understood aspect of lepidopteran biology. 
Importance

Many insects host microbiomes with important ecological functions. However, the

28 prevalence of this phenomenon is unclear, because in many insect taxa microbiomes have only

29 been studied in part of the life cycle, if at all. A prominent example is the butterflies and moths,

30 in which the composition and functional role of adult-stage microbiomes are largely unknown.

31 We comprehensively characterized microbiomes in adult passion-vine butterflies. Butterfly-

32 associated bacterial communities are generally abundant in guts, consistent within populations,

33 and composed of taxa widely shared among hosts. More closely related butterflies harbor more

34 similar microbiomes, with the most dramatic shift in microbiome composition occurring in

35 tandem with a suite of ecological and life history traits unique to the genus Heliconius.

36 Butterflies are also frequently infected with previously undescribed eukaryotic parasites, which

37 may interact with bacteria in important ways. These findings advance our understanding of

38 butterfly biology and of insect-microbe interactions generally.

\section{Introduction}

Insect microbiome research has historically focused on hosts with highly stable, specific,

43 and functionally important microbiomes (1). For example, the obligate nutritional endosymbionts

44 of sap- and blood-feeding insects, which exemplify this scenario, have long served as important

45 model systems. More recently, however, wider microbial explorations have shown that this kind

46 of association does not apply to all insect taxa. In some insect groups, microbiomes are more 
47 variable, and the constituent microbes have low or no specificity to their hosts $(2,3)$. Moreover,

48 the functional importance of these microbiomes is often unclear - and possibly variable, low, or

49 nonexistent in some groups (4). Understanding the nature of microbiomes in these insects is not

50 only important to their biology, but also for a broader understanding of insect microbiome

51 evolution.

52 The Lepidoptera (butterflies and moths) are a group in which microbiome form and

53 function have been particularly difficult to resolve. Recent work indicates that larvae

54 (caterpillars) typically harbor microbiomes that are variable, low-abundance, and transient (5-9).

55 However, a crucial consideration for Lepidoptera and other insects that undergo complete

56 metamorphosis is that microbial associations may change between life stages (10). In

57 Lepidoptera, larvae and adults do often differ in the composition and absolute abundance of their

58 microbiomes $(6,11-15)$. Beyond this pattern, however, the ecology of adult-stage microbiomes

59 remains poorly understood.

There are three major unknowns about microbiomes of adult Lepidoptera that we sought

61 to address in this study. First, it is unclear how variable microbiomes are among host individuals

62 and taxa, and which ecological or evolutionary factors (e.g., host phylogeny) are associated with

63 microbiome variation. Understanding these patterns could give insights into whether

64 microbiomes are important for certain ecological traits, such as feeding, and into the likelihood

65 of host-microbe coevolution. Second, it is unclear whether butterfly-associated microbes have

66 any specificity to their host species or to higher taxonomic levels; tight specificity is a hallmark

67 of obligate insect-microbe symbioses (3). Previous studies of adult Lepidoptera microbiomes

68 have largely used 97\% operational taxonomic units (OTUs) based on short fragments of the 16S

69 rRNA gene. OTUs can encompass substantial strain-level diversity (16), hindering analyses of 
70 specificity. Third, the full diversity of eukaryotic microbes that may be associated with adult

71 Lepidoptera has not been explored, as previous studies have exclusively focused on bacteria or

72 fungi.

Our study focuses on the neotropical passion-vine butterflies (Nymphalidae: Heliconiini).

74 This tribe, which includes the genus Heliconius, is a foundational system in evolutionary biology

75 with over 150 years of scientific study (17). Given the scientific relevance of heliconiines (18),

76 and the myriad roles that microbes can play in animal biology (19), it is important to know the

77 structure and function of heliconiine-associated microbiomes. Heliconiine butterflies are also a

78 potentially useful system for the field of host-microbe interactions, given the wealth of genomic

79 data available (20) and the ability to rear and genetically manipulate members of this group (21).

80 A handful of amplicon sequencing-based microbiome studies have included adult heliconiines,

81 but these were limited to a small number of individuals and species $(11,22,23)$. Consequently,

82 we lack even basic information about the microbes harbored by heliconiine butterflies and how

83 they may vary across individuals and the host phylogeny.

In addition to the practical advantages mentioned above, heliconiines are also an

85 interesting system to address how host ecological traits evolve in concert with microbiomes.

86 Within Heliconiini, the genus Heliconius exhibits unique feeding and life history traits relative to

87 other genera: larvae feed on young passion-vine shoots and leaves as opposed to old foliage,

88 adults of most species (besides $H$. aoede) feed on pollen as well as nectar, and they have

89 distinctive defensive chemistry and a greatly extended adult-stage lifespan of several months (18,

90 24). These traits could influence and be influenced by microbiomes in many ways. For example,

91 we hypothesized that pollen-feeding might be associated with a distinctive microbiome, either

92 because it is a source of microbes (25) or of nutrients that shift resident microbiome composition 
93 (26), or, because the trait itself depends on metabolic contributions from novel microbes (11). A

94 useful starting point to uncovering these kinds of interactions is to examine whether Heliconius

95 have microbiomes that are internally consistent yet distinct from those of other heliconiine

96 genera. If so, then we can zero in on the specific ecological and evolutionary drivers of variation,

97 and the potential functional roles of microbiomes in Heliconius biology.

98 Here we addressed the following main questions: $i$ ) How variable are heliconiine

99 microbiomes among host individuals, species, genera, and across the heliconiine phylogeny? $\mathrm{ii}$ )

100 Are host traits, such as pollen-feeding, associated with microbiome variation? iii) How host-

101 specific are butterfly-associated microbes? At the same time, we sought to facilitate future

102 microbiome research on heliconiine butterflies by answering some additional questions: $i v$ ) How

103 are microbes distributed within the butterfly body? v) How abundant are these microbes? vi)

104 How reliable is amplicon sequencing of 16S rRNA genes for studying butterfly-associated

105 bacterial communities, and vii) are there eukaryotic microbes we might be missing with targeted 106 amplicon sequencing?

107 We collected 214 wild adult butterflies, representing 23 species and subspecies of

108 Heliconiini, and characterized their microbiomes with 16S rRNA gene sequencing. While many

109 adult Lepidoptera microbiome studies have used reared, captive specimens $(6,12,13,22,27)$,

110 these may give a biased picture of microbial community structure in the wild (11). We also used

111 shotgun metagenomic sequencing on a subset of butterflies both to provide an untargeted

112 assessment of microbial diversity (including eukaryotes) and to provide a finer-resolution picture

113 of microbial specificity than is available from amplicon sequencing of short 16S rRNA gene

114 regions. Furthermore, we used quantitative PCR to estimate the absolute abundance of bacteria

115 across different heliconiine taxa and tissue types. Our work illustrates ecological and 
116 evolutionary dynamics of microbiomes in adult heliconiine butterflies and advances our general

117 understanding of Lepidoptera-microbe interactions.

119 Materials and Methods

121 Field collections

The wild adult butterflies used for whole-body microbiome sequencing were collected

124 from seven locations in Panama and Ecuador in May-August 2014 (more detail is provided in the

125 supplemental file "Collection_localities.txt"). Butterflies were euthanized with ethyl acetate and

126 stored in DMSO after removal of wings, following (28). We also stored two DMSO-only blanks

127 to use as negative controls. In June 2016, we collected additional adult butterflies for gut and

128 head/thorax sequencing from Gamboa and Pipeline Road, Panama. For these specimens, we

129 dissected the gut (hindgut, midgut, and the distal $\sim 1 / 2$ of the foregut) using sterilized tools prior

130 to storage in DMSO. The whole head and thorax (including the proximal foregut) were stored

131 separately. Species or subspecies were identified based on morphology. Butterflies were

132 collected under permit \# SC/A-7-11 from Panama's Autoridad Nacional del Ambiente and \#

133 005-13 IC-FAU-DNB/MA from Ecuador's Ministerio del Ambiente.

135 Sample processing, qPCR, PCR and sequencing

We removed whole bodies and head/thorax samples from DMSO and, after

138 homogenization, used approximately $50 \mathrm{mg}$ subsamples of homogenate for DNA extractions 
139 with the MoBio PowerSoil kit following the manufacturer's instructions. We added entire guts

140 directly to DNA extraction tubes, in which they were homogenized during the first bead-beating

141 step of the protocol. Two DMSO blanks and 30 DNA extraction blanks were also processed in

142 tandem with the butterfly samples and sequenced. For dissected gut and head/thorax samples, we

143 estimated total bacterial abundance using quantitative PCR (qPCR) with 16S rRNA gene primers

144 (515F/806R) following the protocol described in ref. (5).

145 PCR amplifications (515F/806R primers, V4 region) and 2 X 150 bp Illumina MiSeq

146 sequencing of 16S rRNA genes followed standard Earth Microbiome Project protocols

147 (dx.doi.org/10.17504/protocols.io.nuudeww). For gut and head/thorax samples of 29 butterfly

148 individuals of nine species, we also attempted PCR amplification with primers that target the ITS

149 gene region of fungi (29). However, amplification success with these fungal-specific primers (as

150 assessed by gel electrophoresis) was very low, suggesting a lack of abundant fungal DNA that

151 was later corroborated with the shotgun metagenomic data (see below). DNA extracts from a

152 subset of 15 amplicon-sequenced gut samples were used for shotgun metagenomic sequencing

153 following the approach described previously (30) with an input DNA concentration of 0.75

154 ng/ul, KAPA HiFi HotStart ReadyMix and bead cleanup with Ampure XP beads at a 0.9x ratio.

Amplicon data processing

Amplicons from the 2014 whole-body samples and 2016 gut and head/thorax samples

159 were sequenced on separate runs, demultiplexed using idemp (https://github.com/yhwu/idemp),

160 and combined for further processing. Cutadapt (31) was used to remove primer sequences. We

161 then used the DADA2 pipeline (32) to quality-filter (max EE value $=1)$ and trim (150 bp 
162 forward, $140 \mathrm{bp}$ reverse) reads, infer exact sequence variants (ESVs), merge paired-end reads,

163 and remove chimeras. We classified ESVs using the RDP Naive Bayesian Classifier algorithm

164 (33) against the SILVA training set v. 132 (34).

165 Further data processing and analyses were conducted in R v. 3.6.0 (35). We used

166 decontam (36) for prevalence-based identification of putative contaminant ESVs based on 34

167 negative controls (DMSO and DNA extraction blanks and PCR no-template controls). The

168 median percentage of contaminant sequences across butterfly samples was $0.09 \%$, but two

169 samples had $>10 \%$ contaminants and were removed from further analysis. ESVs with $<100$ total

170 sequences across all samples (out of a combined total of 5.6 million sequences) were removed,

171 as were ESVs classified as mitochondria or chloroplast, or bacteria lacking sub-domain

172 identification. These ESVs combined typically made up a low proportion of reads from the

173 libraries (median of $2.6 \%$ across all samples). We also used these data to correct qPCR-based

174 absolute abundances for non-bacterial amplification. Specifically, we multiplied the proportion

175 of bacteria in sequence libraries by the total number of 16S rRNA gene copies to obtain

176 estimates of bacterial absolute abundance.

177 As the 16S rRNA gene amplicon libraries were highly variable in read depth across

178 samples, we rarefied all samples to 5,000 sequences, filtering out 13 samples with lower

179 sequence depth. We relabeled Pantoea, Erwinia, Kluyvera, Citrobacter, Klebsiella, and

180 Cronobacter taxonomic assignments to Enterobacter. This step was taken as genera within

181 Enterobacteriaceae are often polyphyletic and are difficult to resolve from short 16S rRNA gene

182 regions (e.g., (37)), and we wanted to avoid spurious separation of ESVs among genera and

183 resulting idiosyncrasies in genus distributions across butterflies. Another case of ambiguous

184 taxonomy occurred with sequences classified as Pseudomonas by the SILVA reference database, 
185 which we label below as "Entomomonas/Pseudomonas". Entomomonas is a recently described

186 genus of apparently insect-specialized Pseudomonadaceae and includes several sequences

187 retrieved from Heliconius genome assemblies (38).

192 UniFrac distances (weighted and unweighted). To obtain a bacterial phylogeny for the latter, we

193 used the fragment-insertion method (39) to place our ESV sequences into the Greengenes

194 reference tree (40). The butterfly phylogeny is from (41) (TreeBASE \#Tr77496). Four of the

195 butterfly species in our sample set contained specimens from two distinct subspecies (e.g.,

196 Heliconius sara sara and Heliconius sara magdalena). To include these in the species-level host

197 phylogeny, we inserted subspecies tips halfway along the terminal branches to their sister

198 subspecies. Hereafter we refer to these subspecies as "species" for simplicity.

200 permutations to calculate the correlation between microbial community dissimilarities/distances

201 and host phylogenetic distances (42). Intraspecific variation in microbiomes was handled by

202 averaging the pairwise dissimilarities/distances between all individuals of one species and all

203 individuals of another species. We used the phytools package (43) to visualize concordance

204 between topologies of the host phylogeny and a dendrogram of bacterial community

205 dissimilarities. Nodes were rotated with the "cophylo" function in phytools to maximize tip

206 matching between the two trees. 
Differences in overall community composition between host genera were tested with

208

209

210

211

212

214

215

216

217

\section{Metagenome data processing and analysis}

For 15 gut samples, we obtained shotgun metagenomic data to complement the bacterial 16S rRNA gene amplicon dataset. We quality-filtered these reads with sickle (47) and trimmed adapters with cutadapt (31). We then used Bowtie 2 (48) to filter out reads matching a given sample's corresponding host species' genome, obtained from Lepbase (20). The two

Dryadula phaetusa metagenomes were mapped to a genome of the sister species Dryas iulia as no Dryadula genome was available. Since there was a high proportion of host-derived reads, we focused here on describing microbial diversity using ribosomal RNA gene reads present in the metagenomes. With the host-filtered reads, we used phyloFlash (49) to find and classify 
230 eukaryotic and bacterial SSU rRNA reads. Bacterial community composition was compared

231 between the amplicon and shotgun metagenomic datasets using a Mantel test. We also used

232 phyloFlash to assemble $16 \mathrm{~S}$ rRNA genes from the $150 \mathrm{bp}$ shotgun reads. These longer sequences

233 allowed us to estimate the phylogeny of Orbus, the dominant bacterium in these 15 samples.

234 Orbus sequences were aligned with MUSCLE (50), curated with Gblocks (51), and used for

235 maximum likelihood reconstruction with the phylogeny.fr implementation (52) of PhyML (53).

237 Data availability

239 Amplicon data, metadata, and R code are available from figshare

240 (figshare.com/projects/Heliconius_butterfly_microbiomes/70520). Metagenomes are available

241 from MG-RAST (project no. MGP89563).

243 Results

Adult heliconiine butterflies host whole-body microbiomes that are typically low in

245 diversity and evenness. Across all individuals, the median ESV richness was 26, of which only

24611 accounted for $95 \%$ or more of the sequences. The composition of these communities is also

247 reasonably consistent within host species. For example, within our most deeply sampled

248 population (H. erato demophoon in Gamboa, Panama; $\mathrm{N}=23$ ), a median of $84 \%$ of the $16 \mathrm{~S}$

249 rRNA gene reads obtained from a given individual's microbiome belonged to a core set of 10

250 bacterial genera (Fig. S1). Some of these genera are present in roughly similar relative

251 abundances across individuals and across our two sampling years (Fig. S1). Applying the

252 aforementioned calculation to all heliconiine species from which five or more individuals were 
sequenced, we found that this intraspecific consistency extends beyond H. erato demophoon. For

254 most host species, over $80 \%$ of the constituent individuals' microbiome reads belonged to the 10

255 most abundant bacterial genera for that species (median $=88 \%$, range $=77-94 \%$ ).

Microbiomes from whole, homogenized butterfly bodies are mainly composed of gut-

257 associated taxa. Isolated guts are similar to conspecific whole-body microbiomes in their

258 bacterial community profiles (Fig. S1), with many bacterial genera occurring at similar relative

259 abundances in guts and head/thorax tissue (Table S1). Some bacteria did differ between whole-

260 body and gut samples, such as Wolbachia, which usually inhabit reproductive tissues (54) (Fig.

261 S1). Likewise, in both Heliconius and other heliconiine genera, the relative abundance of

262 Acinetobacter was $\sim 10$-fold higher in head/thorax samples as compared with guts (Table S1). In

263 contrast, Orbus, Enterobacter, Asaia, and some other dominant bacterial genera are enriched in

264 gut tissue (Table S1).

We used metagenomes $(\mathrm{N}=15)$ to examine the accuracy of amplicon sequencing for

266 describing bacterial community composition in our broader sample set. Interindividual

267 microbiome variation was highly correlated between the amplicon-based dataset and the

268 metagenomic dataset (Mantel $r=0.63, \mathrm{p}<0.001$ ). Furthermore, for six of the most abundant

269 bacterial genera, relative abundances in amplicon libraries were highly predictive of relative

270 abundances in metagenomes (Fig. S2). These results support the use of amplicon sequencing for

271 evaluating bacterial community composition in adult heliconiine butterflies.

Adult Heliconius butterflies generally harbor a high absolute abundance of gut

273 bacteria, though titers sometimes varied substantially even among conspecific individuals

274 collected from the same location (Fig. 1A). On average, Heliconius harbored significantly higher

275 (by $\sim 5.5$-fold) gut bacterial titers than species belonging to other passion-vine butterfly genera (p 
$276=0.039)$. Bacterial communities associated with head and thorax tissue did not significantly

277 differ in titer between Heliconius and other genera ( $\mathrm{p}=0.38$; Fig. S3).

279 compared with other passion-vine butterfly genera (Fig. 1B). Microbiomes clustered by host

280 genera to varying degrees depending on the distance metric used. The effect was strongest with

281 taxonomic or phylogenetic metrics that incorporate information on the relative abundances of

282 exact sequence variants (ESVs) (Bray-Curtis: $\mathrm{R}^{2}=0.13, \mathrm{p}=0.001$; weighted UniFrac: $\mathrm{R}^{2}=0.18$,

$283 \mathrm{p}=0.001)$ as opposed to purely presence/absence-based metrics $\left(\right.$ Jaccard: $\mathrm{R}^{2}=0.11, \mathrm{p}=0.001$;

284 unweighted UniFrac: $\left.\mathrm{R}^{2}=0.10, \mathrm{p}=0.001\right)$. This separation of Heliconius microbiomes from

285 related butterfly genera is not fully explicable by adult-stage diet, as individuals of the non-

286 pollen-feeding species Heliconius aoede mostly clustered with pollen-feeding Heliconius species

287 (Fig. 1B). An analysis of species-by-species Bray-Curtis dissimilarities confirmed that H. aoede

288 microbiomes are not uniquely distinct from those of pollen-feeding Heliconius species (Fig. S4).

microbiome composition was associated with host relatedness (phylogenetic distance). More

291 closely related butterfly lineages harbored more similar microbiomes (Bray-Curtis: Mantel $r=$

$2920.40, \mathrm{p}=0.01$ ), and the topologies of the butterfly phylogeny and the dendrogram of microbiome

293 similarity were moderately congruent (Fig. 2). This pattern was still statistically significant when

294 microbiome variation was measured using weighted UniFrac distances (Mantel $r=0.31, \mathrm{p}=$

295 0.03), but not when using unweighted UniFrac (Mantel $r=0.03, \mathrm{p}=0.41$ ) or Jaccard distances

296 (Mantel $r=0.19, \mathrm{p}=0.08$ ), which do not incorporate relative abundance information.

297 We then determined which bacterial taxa contribute to the observed taxonomic (Fig.

298 1B) and phylogenetic (Fig. 2) structuring in overall community composition. The dominant 
bacterial genera Enterobacter, Orbus, and Entomomonas/Pseudomonas were proportionally

300 more abundant in Heliconius versus the non-pollen-feeding butterfly genera, while Asaia and

301 Apibacter showed the opposite pattern (Fig. 3). None of these bacterial genera, however, were

302 exclusively restricted to one host feeding guild, genus, or species, and their relative abundances

303 were occasionally highly variable (Fig. 3). We also analyzed the data at the ESV-level, including

304 all individual ESVs that were reasonably abundant within at least one host species ( $\geq 5 \%$ within-

305 species average). We did not find evidence for prevalent host species- or genus-restricted ESVs

306 (Fig. 4). Most ESVs are present, albeit with varying relative abundances, across host genus and

307 species boundaries.

Amplicon-derived ESVs are limited in their ability to resolve bacterial strains as they

309 represent only a short region of the $16 \mathrm{~S}$ rRNA gene (here, $\sim 250 \mathrm{bp}$ ). To test for potential host

310 specificity at a finer level of resolution, we obtained near-full-length 16S rRNA gene sequences

311 from the bacterium Orbus, which is highly prevalent across gut and whole-body samples (Fig. 3,

312 Fig. S1) and almost exclusively composed of a single ESV (Fig. 4). These sequences were

313 reconstructed from the 12 metagenomes in which Orbus was sufficiently abundant. A phylogeny

314 based on these sequences and other Orbaceae shows that butterfly-associated Orbus form a

315 unique clade (Fig. 5). Host-phylogenetic or geographic structure was not evident within this

316 clade. In fact, many of the Panamanian heliconiine butterflies harbored Orbus that have identical

317 or near-identical 16S rRNA gene sequences to an Orbus strain isolated from an East Asian

318 butterfly, Sasakia charonda (55).

We also used the shotgun metagenomes to search for microbial eukaryotes in butterfly

320 gut samples and found that microsporidia and trypanosomatids were prevalent (27\% and $47 \%$ of

321 the 15 individual samples analyzed, respectively) (Fig. S5). Fungi and other microeukaryotic 
322 taxa were only sporadically detected, and at very low relative abundances. When

323 trypanosomatids were detected in a given sample, we also sometimes detected 16S rRNA gene

324 reads in the metagenomic data classified as Kinetoplastibacterium, an obligate bacterial

325 endosymbiont of certain trypanosomatids (56). Kinetoplastibacterium was not detected without

326 its corresponding trypanosomatid host (Fig. S5). This observation led us to reexamine the larger,

327 amplicon-based bacterial dataset. We found that $9 \%$ of Heliconius individuals and $4 \%$ of

328 individuals belonging to other heliconiine genera were infected with a trypanosomatid, as

329 inferred by the presence of Kinetoplastibacterium. These proportions were not significantly

330 different (Fisher's Exact Test, $\mathrm{p}=0.28$ ). They are also likely underestimates, given the

331 aforementioned prevalence of trypanosomatids among metagenomes (47\%) and the potential for

332 false negatives - in five metagenomes, trypanosomatids were detected without

333 Kinetoplastibacterium (Fig. S5).

335 Discussion

We found that adult-stage microbiomes in heliconiine butterflies are generally similar in

338 composition among conspecific individuals and over time, and are also abundant within gut

339 tissue (especially in Heliconius). Our estimates of total bacterial abundances (via qPCR), in

340 parallel with those of ref. (23), support the hypothesis that bacteria actively colonize adult

341 butterflies as opposed to passing through the gut transiently. These features contrast with the

342 typical situation in lepidopteran larvae (5), reinforcing the idea that insect-microbe associations

343 can differ strongly between life stages (10). 
Although stable relative to larvae, dominant butterfly-associated microbes do exhibit

345 higher levels of interindividual variability as compared with obligate nutritional endosymbionts

346 or gut microbes of some other insect groups. For example, in honey bees, $>98 \%$ of sequences

347 belong to a honey bee-specific set of 5-9 bacterial species (57). We found that $\sim 80-90 \%$ of

348 sequences in a given butterfly species' microbiome belonged to a set of 10 core bacterial genera.

349 This contrast suggests that microbiomes are more facultative for butterflies or that there is

350 greater functional redundancy among butterfly-associated microbes.

$351 \quad$ We also discovered that Heliconius butterfly microbiomes are distinct from those of other

352 heliconiine genera, in terms of overall community structure (Fig. 1B), relative abundances of

353 specific bacterial genera (Fig. 3), and total numbers of gut bacteria (Fig. 1A). This finding opens

354 the possibility that one or more of the ecological traits specific to Heliconius influences, and may

355 be influenced by, the microbiome. As these traits evolved in tandem (18) it is difficult to

356 disentangle their potential links to microbes, but our analysis of the species Heliconius aoede

357 suggests that pollen-feeding is not a primary driver. $H$. aoede does not pollen-feed $(58,59)$, yet

358 its microbiomes were not uniquely distinct from pollen-feeding Heliconius species (Fig. 1B, Fig.

359 S4). This result contrasts with previous work on butterflies finding a strong association between

360 adult-stage feeding ecology (nectivory versus frugivory) and adult gut microbiomes (23).

361 Larval feeding ecology, however, warrants further investigation as a potential driver of

362 variation in adult gut microbiomes. Microbiomes of butterfly larvae and adults are not fully

363 decoupled: in H. erato, some adult-stage microbes appear to be carried over from the larval stage

364 (11). All Heliconius (including H. aoede) feed on young passion-vine foliage while other

365 heliconiine genera feed on mature foliage (18). Related variation in diet-derived microbes, or in 
366 host processes that determine which microbes persist through metamorphosis $(10,60)$, could

367 potentially underlie variation in adult-stage microbiomes.

368 In addition to the clear separation of Heliconius from other heliconiine genera (Fig.

369 1B), there was also a strong signal of the host phylogeny in heliconiine butterfly microbiomes

370 (Fig. 2). The strength of this signal was comparable to that observed in gut microbiomes of

371 mammals (61) and other host groups (42). This result is also in agreement with a recent survey of

372 neotropical butterflies, in which microbiomes were found to be phylogenetically structured

373 across a broad diversity of hosts (six different families) (23). Thus, even in a comparatively

374 young radiation such as the one studied here (41), host phylogenetic history is clearly an

375 important factor shaping the composition of adult butterfly microbiomes. The question then

376 becomes, how has this pattern (also known as phylosymbiosis (62)) arisen, and what does it

377 signify?

There are at least two, non-mutually exclusive processes that can lead to

379 phylosymbiosis: host-microbe co-diversification, and contemporary host filtering of

380 environmental microbes $(42,63)$. In the former process, there is a shared evolutionary history of

381 host and microbial lineages. This does not necessarily apply to the latter process, which occurs

382 when host traits that influence microbial colonization, such as diet preference, are conserved to

383 some degree across the host phylogeny. We suggest that co-diversification is unlikely in this

384 system given the broad distribution of bacterial phylotypes across host species and genera

385 (discussed below). Rather, host filtering of environmental microbes likely explains phylogenetic

386 structure in heliconiine microbiomes, as is the case in a wide variety of other animal groups (42).

387 A priority for future work on butterflies is to identify the specific traits underlying host filtering.

388 In Heliconiini, two candidates are larval host plant use (i.e., Passiflora species identity and age 
of tissue consumed) and adult foraging behavior (especially whether pollen is collected, and from which plant species), both of which exhibit phylogenetic signal $(18,64,65)$. was largely driven by shifts in the relative abundance of shared bacterial genera and ESVs, as opposed to the presence or absence of host-specific bacterial taxa (Figs. 3, 4). Moreover, a finer-

394 resolution analysis of the nearly ubiquitous bacterium Orbus did not find evidence for specificity within butterflies (Fig. 5). This pattern is notable in part because it weighs against the codiversification model described above, and in part because it provides a contrast to the high degree of host specificity documented in a number of other insect-microbe symbioses (66-69). While not highly host-specific, at least some of the dominant bacteria in butterflies appear to be insect specialists as opposed to cosmopolitan environmental taxa. For example, phylogenetic evidence supports a general insect association for Entomomonas (38), Apibacter (70), and Orbus

401 (Fig. 5) (71). The ecology of these groups is poorly understood; one important unknown is how they are transmitted among butterflies, which may be via flowers (72) or other shared resources. in gut tissue of many adult heliconiine butterflies (Fig. S5). Beyond fungi $(12,23)$, which do not appear to be abundant in heliconiines, microeukaryotes are almost unknown from adult-stage

407 Lepidoptera - with the exception of the neogregarine Ophryocystis elektroscirrha in monarch

408 butterflies (73). Given their potential interactions with gut bacteria and relevance to host fitness, 409 more targeted analyses of butterfly-associated microeukaryote diversity are clearly warranted. While effects of endosymbionts such as Wolbachia and Spiroplasma have been

411 documented (74), a major open question is what functional role gut microbes play in the biology 
412 of adult heliconiines and Lepidoptera generally (75). In Heliconius, our hypothesis that microbes

413 mediate pollen-feeding is not strongly supported. To directly influence pollen digestion-which

414 occurs extraorally using saliva exuded from the proboscis (76) — microbes would likely need to

415 inhabit the proboscis or salivary gland. Yet we saw no clear signal of elevated microbial

416 abundances or unique microbial taxa in these tissues in Heliconius as compared with non-pollen-

417 feeding butterflies (Fig. S3). Endogenous pollen-digestion mechanisms $(59,77,78)$ may be

418 sufficient.

419 Other microbial roles in host nutrition are possible, although a recent experiment on the

420 butterfly Speyeria mormonia did not find evidence for nutrition-related functions (79). We

421 suggest that colonization resistance (i.e., protection from pathogens and parasites), which is a

422 feature common to many symbioses $(63,80)$, could be a primary ecological function of adult

423 butterfly gut microbiomes. We found that heliconiines are frequently infected by

424 trypanosomatids and microsporidia, as well as Serratia (Fig. 4), which are common opportunistic

425 bacterial pathogens in insects $(81,82)$. Adult butterflies may be similar to pyrrhocorid bugs,

426 tsetse flies and social bees, in which gut bacteria provide an important layer of defense against

427 related parasites and pathogens (83-87). Importantly, colonization resistance can readily evolve

428 even in symbioses lacking strong host-microbe specificity (88), such as that between heliconiines

429 and their adult-stage microbiomes.

\section{Conclusions}


435 biology. The characteristics of adult-stage microbiomes we report contrast with larval

436 Lepidoptera, emphasizing that holometabolous insects are able to interact with microbes in very

437 different ways across life stages (10). However, many Lepidoptera do not feed or even have a

438 digestive tract as adults (89), and we do not yet know whether patterns from heliconiines or other

439 butterflies are generalizable to these other groups. We further suggest that heliconiines may serve

440 as a useful system for exploring how animal ecology and life history relate to microbes.

441 Heliconius host phylogenetically structured microbiomes that are markedly distinct from those of

442 their close relatives, and this finding sets the stage for experiments to test the specific host traits

443 that may be involved. Finally, we uncovered novel microeukaryote diversity in butterflies, and

444 hypothesize that they are parasites that interact antagonistically with gut bacteria. Further

445 research on adult-stage microbiomes will help advance our understanding of both insect-

446 microbiome evolution and of the biology of Lepidoptera, a diverse group of considerable

447 ecological, societal, and scientific importance $(89,90)$.

449 Acknowledgments

This material is based on work supported by the Cooperative Institute for Research in

452 Environmental Sciences, the Ecology and Evolutionary Biology Department at the University of

453 Colorado Boulder, a National Science Foundation Graduate Research Fellowship (1144083), and

454 a postdoctoral fellowship from the USDA National Institute of Food and Agriculture (2018-

455 08156), to TJH. This work was further supported by grants from the Simons Foundation

456 (429440) and by funding from the Smithsonian Tropical Research Institute. We thank Jessica

457 Henley for assistance with sequencing, the Smithsonian Tropical Research Institute staff for 
458 administrative support and the Panamanian and Ecuadorian Authorities for permission to collect

459 butterflies. We also acknowledge the reviewers for helpful comments that improved the

460 manuscript.

461

462 References

463

464 1. Buchner P. 1965. Endosymbiosis of animals with plant microorganisms. John Wiley \& 465 Sons.

466 2. Jones RT, Sanchez LG, Fierer N. 2013. A Cross-Taxon Analysis of Insect-Associated 467 Bacterial Diversity. PLoS ONE 8:e61218.

468 3. Engel P, Moran NA. 2013. The gut microbiota of insects - diversity in structure and 469 function. FEMS Microbiology Reviews 37:699-735.

470 4. Hammer TJ, Sanders JG, Fierer N. 2019. Not all animals need a microbiome. FEMS

$471 \quad$ Microbiology Letters 366:fnz117.

472 5. Hammer TJ, Janzen DH, Hallwachs W, Jaffe SP, Fierer N. 2017. Caterpillars lack a 473 resident gut microbiome. Proceedings of the National Academy of Sciences 114:96414749646.

475 6. Staudacher H, Kaltenpoth M, Breeuwer JAJ, Menken SBJ, Heckel DG, Groot AT. 2016.

476 Variability of bacterial communities in the moth Heliothis virescens indicates transient $477 \quad$ association with the host. PLoS ONE 11:e0154514. 
478 7. Priya NG, Ojha A, Kajla MK, Raj A, Rajagopal R. 2012. Host Plant Induced Variation in

$479 \quad$ Gut Bacteria of Helicoverpa armigera. PLoS ONE 7:e30768.

480 8. Whitaker M, Pierce N, Salzman S, Kaltenpoth M, Sanders J. 2016. Microbial communities 481 of lycaenid butterflies do not correlate with larval diet. Frontiers in Microbiology 7:1920.

482 9. Phalnikar K, Kunte K, Agashe D. 2019. Disrupting butterfly caterpillar microbiomes does 483 not impact their survival and development. Proc R Soc B 286:20192438.

484 10. Hammer TJ, Moran NA. 2019. Links between metamorphosis and symbiosis in 485 holometabolous insects. Phil Trans R Soc B 374:20190068.

486 11. Hammer TJ, McMillan WO, Fierer N. 2014. Metamorphosis of a Butterfly-Associated 487 Bacterial Community. PLoS ONE 9:e86995.

488 12. Harrison JG, Urruty DM, Forister ML. 2016. An exploration of the fungal assemblage in 489 each life history stage of the butterfly, Lycaeides melissa (Lycaenidae), as well as its host $490 \quad$ plant Astragalus canadensis (Fabaceae). Fungal Ecology 22:10-16.

491 13. Paniagua Voirol LR, Weinhold A, Johnston PR, Fatouros NE, Hilker M. 2020. Legacy of a 492 Butterfly's Parental Microbiome in Offspring Performance. Appl Environ Microbiol $493 \quad 86: \mathrm{e} 00596-20$.

494 14. Phalnikar K, Kunte K, Agashe D. 2018. Dietary and developmental shifts in butterfly495 associated bacterial communities. Royal Society Open Science 5:171559. 
15. Wang X, Sun S, Yang X, Cheng J, Wei H, Li Z, Michaud JP, Liu X. 2020. Variability of Gut Microbiota Across the Life Cycle of Grapholita molesta (Lepidoptera: Tortricidae). Front Microbiol 11:1366.

16. Chase AB, Karaoz U, Brodie EL, Gomez-Lunar Z, Martiny AC, Martiny JBH. 2017. Microdiversity of an Abundant Terrestrial Bacterium Encompasses Extensive Variation in Ecologically Relevant Traits. mBio 8:e01809-17.

17. Bates HW. 1861. Contributions to an Insect Fauna of the Amazon Valley. Lepidoptera: Heliconidae. Transactions of the Linnean Society of London 23:495-566.

18. Jiggins CD. 2016. The ecology and evolution of Heliconius butterflies. Oxford University Press.

19. McFall-Ngai M, Hadfield MG, Bosch TCG, Carey HV, Domazet-Lošo T, Douglas AE, Dubilier N, Eberl G, Fukami T, Gilbert SF, Hentschel U, King N, Kjelleberg S, Knoll AH,

20. Challis RJ, Kumar S, Dasmahapatra KK, Jiggins CD, Blaxter M. 2016. Lepbase: the a new imperative for the life sciences. Proceedings of the National Academy of Sciences Vargas M, Sanjeev M, Morrison C, Tian D, Aguirre P, Ferrara S, Foley J, Pardo-Diaz C, 
A, McMillan WO. 2019. Interplay between Developmental Flexibility and Determinism in the Evolution of Mimetic Heliconius Wing Patterns. Current Biology 29:3996-4009.e4. among young Heliconius butterfly species. PeerJ 6:e5502.

23. Ravenscraft A, Berry M, Hammer T, Peay K, Boggs C. 2019. Structure and function of the bacterial and fungal gut microbiota of Neotropical butterflies. Ecological Monographs 89:e01346. Schnell S. 2016. Bacterial microbiota associated with flower pollen is influenced by pollination type, and shows a high degree of diversity and species-specificity: The bacterial structure differs between honeybees in winter and summer. ISME J 14:801-814. monarch butterfly (Danaus plexippus). Journal of Invertebrate Pathology 20:51-58. 
537 29. Gardes M, Bruns TD. 1993. ITS primers with enhanced specificity for basidiomycetes -

538 application to the identification of mycorrhizae and rusts. Mol Ecol 2:113-118.

539 30. Baym M, Kryazhimskiy S, Lieberman TD, Chung H, Desai MM, Kishony R. 2015.

540 Inexpensive Multiplexed Library Preparation for Megabase-Sized Genomes. PLOS ONE

$541 \quad 10: \mathrm{e} 0128036$.

542 31. Martin M. 2011. Cutadapt removes adapter sequences from high-throughput sequencing

543 reads. EMBnet.journal 17:10-12.

544 32. Callahan BJ, McMurdie PJ, Rosen MJ, Han AW, Johnson AJA, Holmes SP. 2016.

545 DADA2: High-resolution sample inference from Illumina amplicon data. Nat Methods

$546 \quad 13: 581-583$.

547 33. Wang Q, Garrity GM, Tiedje JM, Cole JR. 2007. Naive Bayesian classifier for rapid

548 assignment of rRNA sequences into the new bacterial taxonomy. Applied and

$549 \quad$ Environmental Microbiology 73:5261-5267.

550 34. Quast C, Pruesse E, Yilmaz P, Gerken J, Schweer T, Yarza P, Peplies J, Glöckner FO.

551 2012. The SILVA ribosomal RNA gene database project: improved data processing and

552 web-based tools. Nucleic Acids Research 41:D590-D596.

553 35. R Core Team. 2016. R: A language and environment for statistical computing. R

$554 \quad$ Foundation for Statistical Computing, Vienna, Austria. 
36. Davis NM, Proctor DM, Holmes SP, Relman DA, Callahan BJ. 2018. Simple statistical identification and removal of contaminant sequences in marker-gene and metagenomics data. Microbiome 6:226.

37. Brady C, Cleenwerck I, Venter S, Coutinho T, De Vos P. 2013. Taxonomic evaluation of the genus Enterobacter based on multilocus sequence analysis (MLSA). Systematic and Applied Microbiology 36:309-319.

38. Wang J, Su Q, Zhang X, Li C, Luo S, Zhou X, Zheng H. 2020. Entomomonas moraniae gen. nov., sp. nov., a member of the family Pseudomonadaceae isolated from Asian honey bee gut, possesses a highly reduced genome. International Journal of Systematic and Evolutionary Microbiology 70:165-171.

39. Janssen S, McDonald D, Gonzalez A, Navas-Molina JA, Jiang L, Xu ZZ, Winker K, Kado DM, Orwoll E, Manary M, Mirarab S, Knight R. 2018. Phylogenetic Placement of Exact Amplicon Sequences Improves Associations with Clinical Information. mSystems

40. McDonald D, Price MN, Goodrich J, Nawrocki EP, DeSantis TZ, Probst A, Andersen GL, Knight R, Hugenholtz P. 2012. An improved Greengenes taxonomy with explicit ranks for ecological and evolutionary analyses of bacteria and archaea. The ISME Journal 6:610_ Butterflies. Systematic Biology 64:505-524. 
42. Mazel F, Davis KM, Loudon A, Kwong WK, Groussin M, Parfrey LW. 2018. Is Host Filtering the Main Driver of Phylosymbiosis across the Tree of Life? mSystems 3:e0009718.

43. Revell LJ. 2012. phytools: An R package for phylogenetic comparative biology (and other things). Methods in Ecology and Evolution 3:217-223.

44. Oksanen J, Kindt R, Legendre P. 2019. vegan: Community Ecology Package. R package version 2.5-6.

45. Anderson MJ, Walsh DCI. 2013. PERMANOVA, ANOSIM, and the Mantel test in the face of heterogeneous dispersions: What null hypothesis are you testing? Ecological Monographs 83:557-574.

46. Pinheiro J, Bates D, DebRoy S, Sarkar D, R Core Team. 2019. Linear and Nonlinear Mixed Effects Models.

47. Joshi, NA, Fass, JN. Sickle: A sliding-window, adaptive, quality-based trimming tool for FastQ files. https://github.com/najoshi/sickle.

48. Langmead B, Salzberg SL. 2012. Fast gapped-read alignment with Bowtie 2. Nat Methods $9: 357-359$.

49. Gruber-Vodicka HR, Seah BK, Pruesse E. 2019. phyloFlash — Rapid SSU rRNA profiling and targeted assembly from metagenomes. bioRxiv https://doi.org/10.1101/521922.

594 50. Edgar RC. 2004. MUSCLE: Multiple sequence alignment with high accuracy and high throughput. Nucleic Acids Research 32:1792-1797. 
51. Castresana J. 2000. Selection of Conserved Blocks from Multiple Alignments for Their Use in Phylogenetic Analysis. Molecular Biology and Evolution 17:540-552.

52. Dereeper A, Guignon V, Blanc G, Audic S, Buffet S, Chevenet F, Dufayard J-F, Guindon S, Lefort V, Lescot M, Claverie J-M, Gascuel O. 2008. Phylogeny.fr: robust phylogenetic analysis for the non-specialist. Nucleic Acids Research 36:W465-W469.

53. Guindon S, Delsuc F, Dufayard JF, Gascuel O. 2009. Estimating maximum likelihood phylgenies with PhyML. Methods in Molecular Biology 537:113-137.

54. Saridaki A, Bourtzis K. 2010. Wolbachia: more than just a bug in insects genitals. Current Opinion in Microbiology 13:67-72. Bae J-W. 2012. Orbus sasakiae sp. nov., a bacterium isolated from the gut of the butterfly Sasakia charonda, and emended description of the genus Orbus. International Journal of Systematic and Evolutionary Microbiology 63:1766-1770.

56. De Souza W, Motta MCM. 1999. Endosymbiosis in protozoa of the Trypanosomatidae family. FEMS Microbiology Letters 173:1-8. gut microbiota research. Lab Animal 47:317-325.

613 58. Brown KS. 1981. The Biology of Heliconius and Related Genera. Annual Review of $614 \quad$ Entomology 26:427-456. 
615 59. Gilbert LE. 1972. Pollen Feeding and Reproductive Biology of Heliconius Butterflies.

616 Proceedings of the National Academy of Sciences 69:1403-1407.

617 60. Johnston PR, Rolff J. 2015. Host and Symbiont Jointly Control Gut Microbiota during

$618 \quad$ Complete Metamorphosis. PLOS Pathogens 11:e1005246.

619 61. Groussin M, Mazel F, Sanders JG, Smillie CS, Lavergne S, Thuiller W, Alm EJ. 2017.

620 Unraveling the processes shaping mammalian gut microbiomes over evolutionary time.

$621 \quad$ Nature Communications 8:14319.

622 62. Lim SJ, Bordenstein SR. 2020. An introduction to phylosymbiosis. Proc R Soc B

$623 \quad 287: 20192900$.

624 63. Moran NA, Ochman H, Hammer TJ. 2019. Evolutionary and Ecological Consequences of 625 Gut Microbial Communities. Annu Rev Ecol Evol Syst 50:451-475.

626 64. Estrada C, Jiggins CD. 2002. Patterns of pollen feeding and habitat preference among $627 \quad$ Heliconius species. Ecological Entomology 27:448-456.

628 65. Boggs CL, Smiley JT, Gilbert LE. 1981. Patterns of pollen exploitation by Heliconius

629 butterflies. Oecologia 48:284-289.

630 66. Ohkuma M. 2008. Symbioses of flagellates and prokaryotes in the gut of lower termites.

631 Trends in Microbiology 16:345-352.

632 67. Kikuchi Y, Hosokawa T, Nikoh N, Meng X-Y, Kamagata Y, Fukatsu T. 2009. Host-

633 symbiont co-speciation and reductive genome evolution in gut symbiotic bacteria of

634 acanthosomatid stinkbugs. BMC Biol 7:2. 
635 68. Kwong WK, Moran NA. 2015. Evolution of host specialization in gut microbes: The bee

636 gut as a model. Gut Microbes 6:214-220.

637 69. Kaltenpoth M, Roeser-Mueller K, Koehler S, Peterson A, Nechitaylo TY, Stubblefield JW,

638 Herzner G, Seger J, Strohm E. 2014. Partner choice and fidelity stabilize coevolution in a

639 Cretaceous-age defensive symbiosis. Proceedings of the National Academy of Sciences

$640 \quad 111: 6359-64$.

641 70. Kwong WK, Moran NA. 2016. Apibacter adventoris gen. nov., sp. nov., a member of the

642 phylum Bacteroidetes isolated from honey bees. International Journal of Systematic and

643 Evolutionary Microbiology 66:1323-1329.

644 71. Martinson VG, Carpinteyro-Ponce J, Moran NA, Markow TA. 2017. A Distinctive and

645 Host-Restricted Gut Microbiota in Populations of a Cactophilic Drosophila Species.

$646 \quad$ Applied and Environmental Microbiology 83:e01551-17.

647 72. Graystock P, Goulson D, Hughes WOH. 2015. Parasites in bloom: flowers aid dispersal and

648 transmission of pollinator parasites within and between bee species. Proceedings of the

$649 \quad$ Royal Society B: Biological Sciences 282:20151371.

650 73. Altizer SM, Oberhauser KS. 1999. Effects of the Protozoan Parasite Ophryocystis

651 elektroscirrha on the Fitness of Monarch Butterflies (Danaus plexippus). Journal of

$652 \quad$ Invertebrate Pathology 74:76-88.

653 74. Duplouy A, Hornett EA. 2018. Uncovering the hidden players in Lepidoptera biology: the 654 heritable microbial endosymbionts. PeerJ 6:e4629. 
655 75. Paniagua Voirol LR, Frago E, Kaltenpoth M, Hilker M, Fatouros NE. 2018. Bacterial

656 Symbionts in Lepidoptera: Their Diversity, Transmission, and Impact on the Host. Frontiers

657 in Microbiology 9:556.

658 76. Eberhard SH, Hikl AL, Boggs CL, Krenn HW. 2009. Saliva or Regurgitated Nectar? What

659 Heliconius Butterflies (Lepidoptera: Nymphalidae) Use for Pollen Feeding. Annals of the

$660 \quad$ Entomological Society of America 102:1105-1108.

661 77. Harpel D, Cullen DA, Ott SR, Jiggins CD, Walters JR. 2015. Pollen feeding proteomics:

662 Salivary proteins of the passion flower butterfly, Heliconius melpomene. Insect

663 Biochemistry and Molecular Biology 63:7-13.

664 78. Smith G, Macias-Muñoz A, Briscoe AD. 2016. Gene Duplication and Gene Expression

665 Changes Play a Role in the Evolution of Candidate Pollen Feeding Genes in Heliconius

666 Butterflies. Genome Biology and Evolution 8:2581-2596.

667 79. Ravenscraft A, Kish N, Peay K, Boggs C. 2019. No evidence that gut microbiota impose a 668 net cost on their butterfly host. Molecular Ecology 28:2100-2117.

669 80. Kamada N, Chen GY, Inohara N, Núñez G. 2013. Control of pathogens and pathobionts by 670 the gut microbiota. Nature Immunology 14:685-690.

671 81. Raymann K, Coon KL, Shaffer Z, Salisbury S, Moran NA. 2018. Pathogenicity of Serratia 672 marcescens Strains in Honey Bees. mBio 9:e01649-18. 
673 82. Grimont F, Grimont PAD. 2006. The Genus Serratia, p. 219-244. In Dworkin, M, Falkow,

674 S, Rosenberg, E, Schleifer, K-H, Stackebrandt, E (eds.), The Prokaryotes. Springer New

675 York.

676 83. Onchuru TO, Martinez AJ, Kaltenpoth M. 2018. The cotton stainer's gut microbiota 677 suppresses infection of a cotransmitted trypanosomatid parasite. Molecular Ecology $678 \quad 27: 3408-3419$.

679 84. Raymann K, Shaffer Z, Moran NA. 2017. Antibiotic exposure perturbs the gut microbiota and elevates mortality in honeybees. PLoS Biology 15:e2001861.

85. Koch H, Schmid-Hempel P. 2011. Socially transmitted gut microbiota protect bumble bees against an intestinal parasite. Proceedings of the National Academy of Sciences 108:1928819292.

86. Weiss BL, Maltz MA, Vigneron A, Wu Y, Walter KS, O’Neill MB, Wang J, Aksoy S. 2019. Colonization of the tsetse fly midgut with commensal Kosakonia cowanii Zambiae inhibits trypanosome infection establishment. PLOS Pathogens 15:e1007470.

87. Wu Y, Zheng Y, Chen Y, Chen G, Zheng H, Hu F. 2020. Apis cerana gut microbiota contribute to host health though stimulating host immune system and strengthening host resistance to Nosema ceranae. R Soc Open Sci 7:192100. advantage provided by the microbiome. Phil Trans R Soc B 375:20190592. 
bioRxiv preprint doi: https://doi.org/10.1101/820829; this version posted August 14,2020 . The copyright holder for this preprint (which was not certified by peer review) is the author/funder, who has granted bioRxiv a license to display the preprint in perpetuity. It is made available under aCC-BY-NC-ND 4.0 International license.

693 90. Boggs CL, Watt WB, Ehrlich PR. 2003. Butterflies: ecology and evolution taking flight.

694 The University of Chicago Press. 
Figures

697

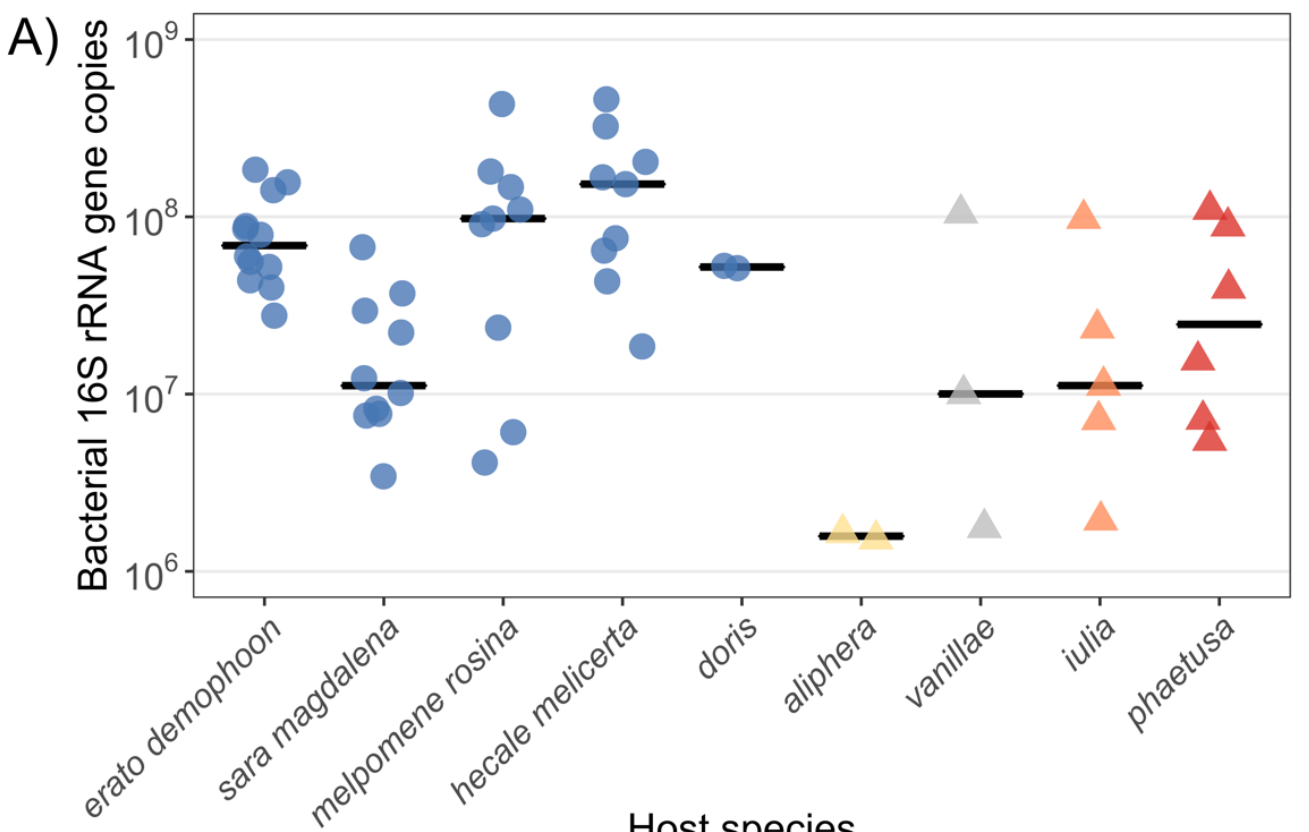

B)

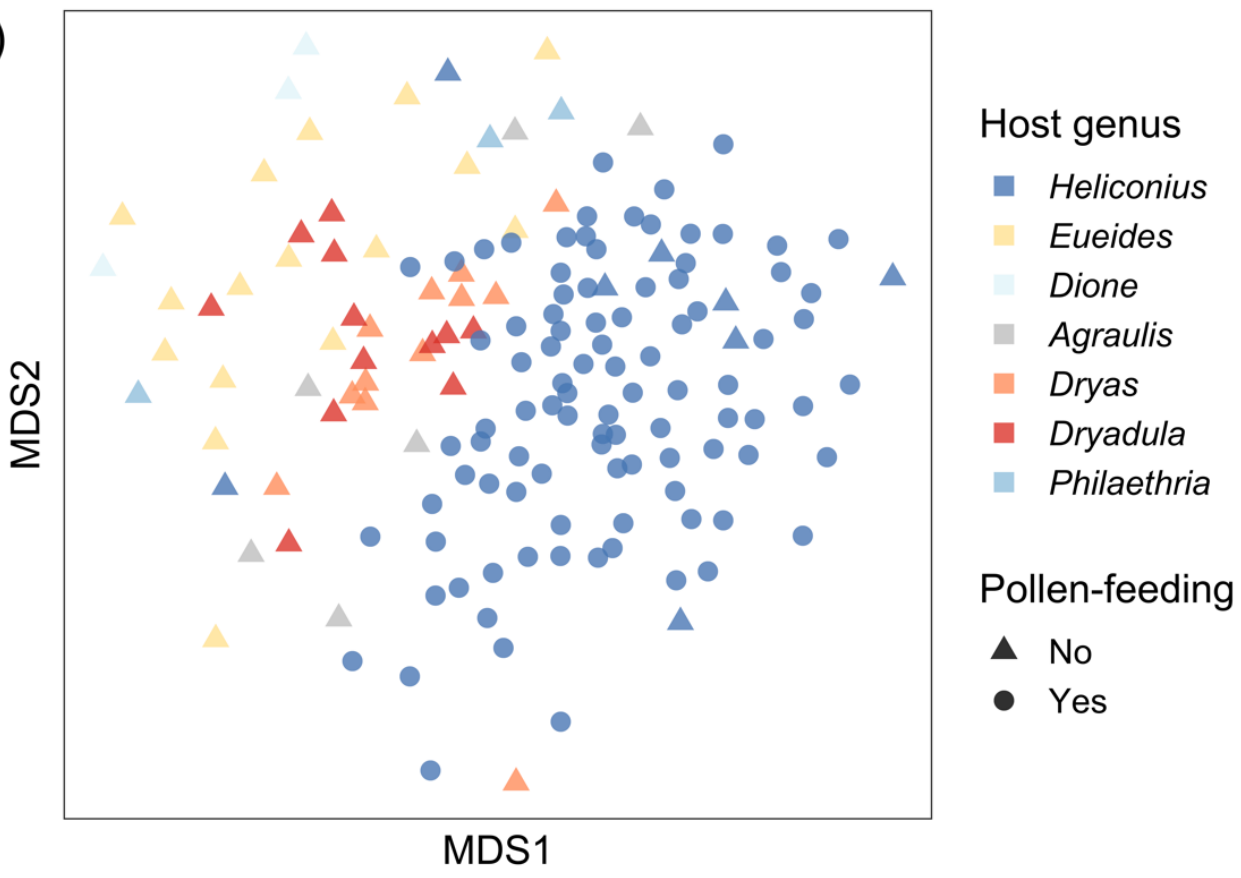

Figure 1. A) Adult Heliconius butterflies have high, though often variable, titers of gut bacteria. Shown are quantitative PCR-derived estimates of bacterial abundances in terms of the number of bacterial 16S rRNA gene copies per individual gut. These individuals were collected from Gamboa, Panama in 2016 (N $=42$ Heliconius, 16 other Heliconiini). B) Heliconius host distinct adult-stage bacterial communities compared with related genera. Shown is an ordination of microbiome variation (Bray-Curtis dissimilarities) among all whole-body samples collected at various sites in Panama and Ecuador in 2014 $(\mathrm{N}=104$ Heliconius, 52 other Heliconiini). With the exception of $H$. aoede (dark blue triangles), pollen- 
bioRxiv preprint doi: https://doi.org/10.1101/820829; this version posted August 14,2020 . The copyright holder for this preprint (which was not certified by peer review) is the author/funder, who has granted bioRxiv a license to display the preprint in perpetuity. It is made available under aCC-BY-NC-ND 4.0 International license.

\section{Host phylogeny}

\section{Microbiome dendrogram}

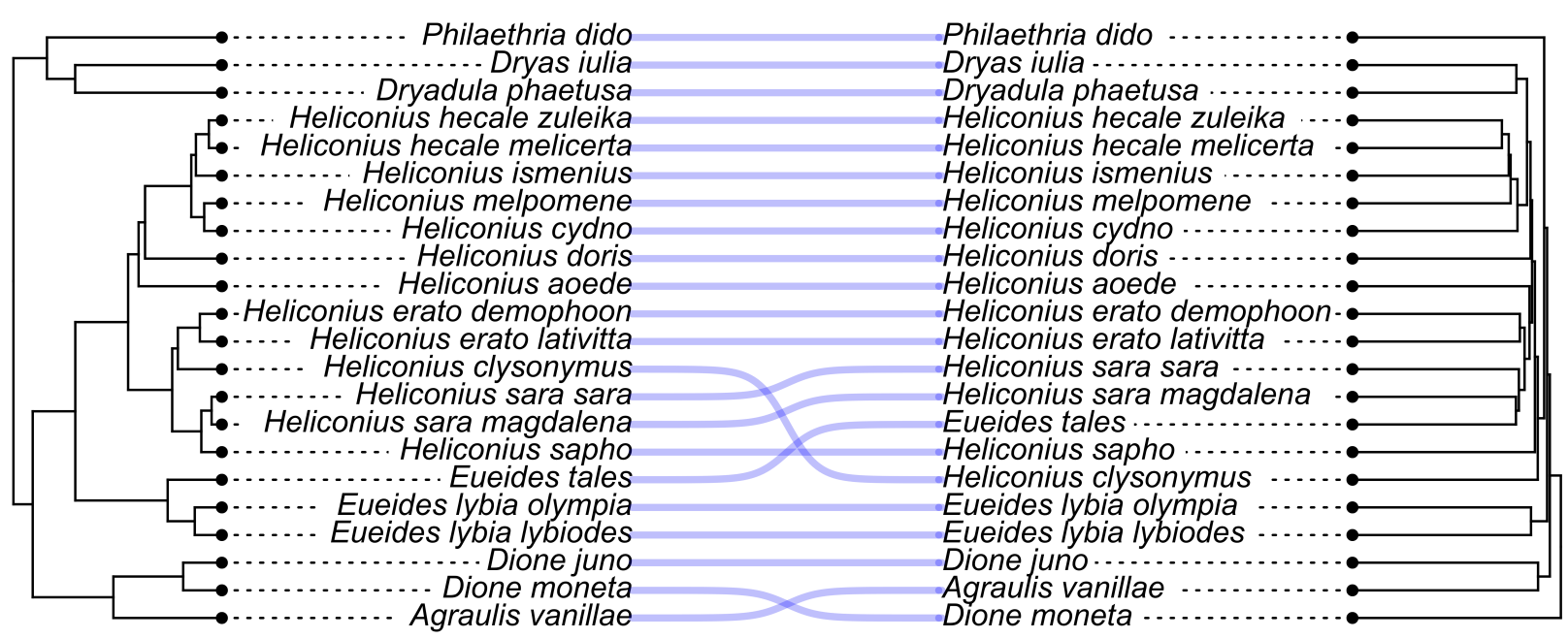

Figure 2. Variation in heliconiine butterfly microbiomes is correlated with host relatedness. Shown is the concordance between the host phylogeny (from (41)) and a dendrogram representation of microbiome variation among species (Bray-Curtis dissimilarities). Here, all nodes have been rotated to maximize tip matching. Note, however, that sets of parallel lines connecting tips between the phylogeny and the dendrogram do not always signify congruent branching structure. 


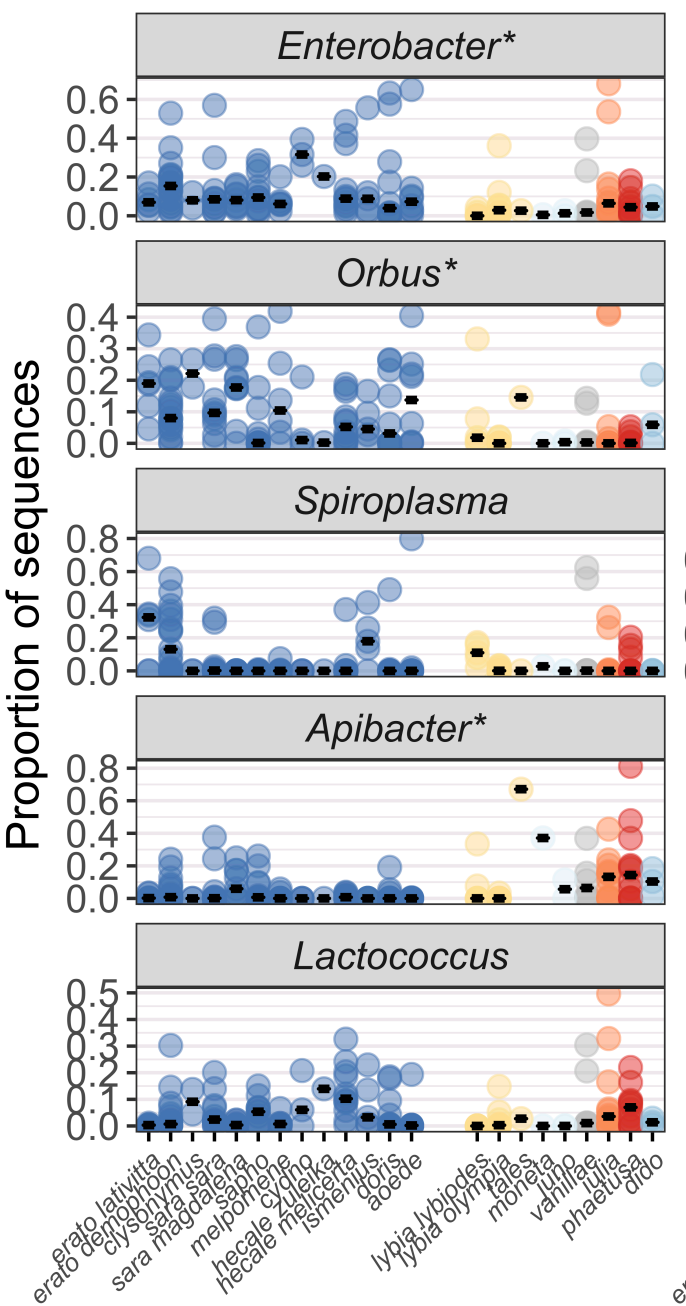

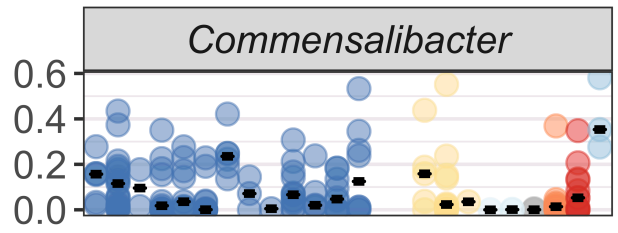
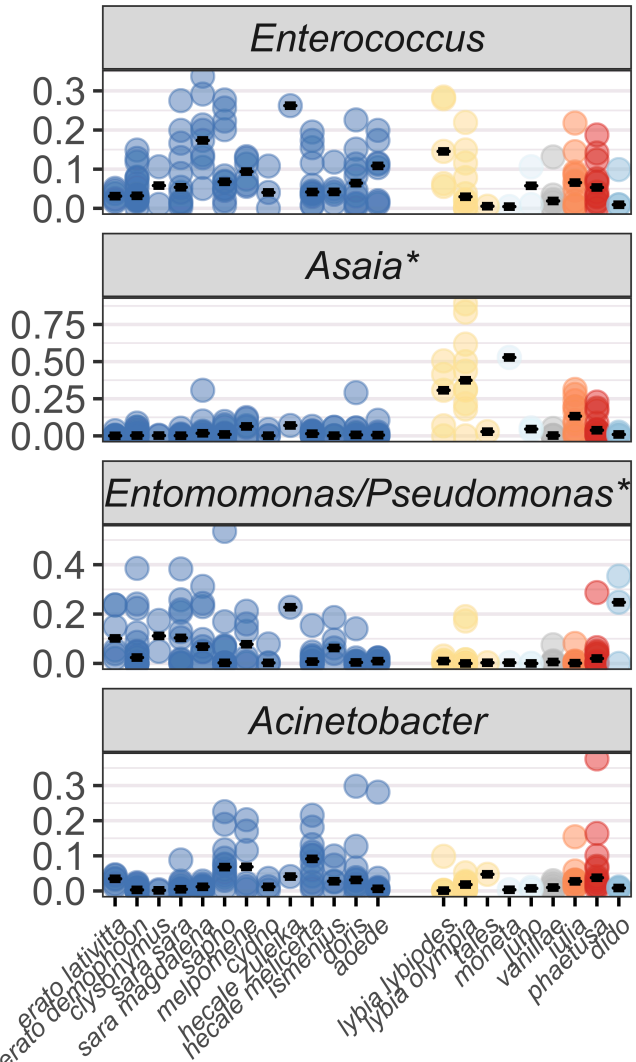

Host genus

Heliconius

Eueides

Dione

Agraulis

Dryas

Dryadula

Philaethria

\section{Host species}

Figure 3. Dominant bacterial genera are largely shared among Heliconius and other butterflies, although some are differentially abundant. Shown are the relative abundances of the top 10 bacterial genera, ranked by mean abundance, in whole-body microbiomes. Dots indicate replicate individuals, and black bars indicate median proportions within a host species. Starred bacterial genera differed significantly in relative abundance between Heliconius and non-Heliconius butterflies ( $<0.05$ after FDR correction). The arrangement of host species on the $\mathrm{x}$ axis corresponds to the phylogeny shown in Fig. 4. Note that Enterobacter here includes sequences originally assigned as Klebsiella and some other closely related Enterobacteriaceae genera (see Methods). 
bioRxiv preprint doi: https://doi org/10.1101/820829; this version posted August 14,2020 . The copyright holder for this preprint (which was not certified by peer review) is the author/funder, who has granted bioRxiv a license to display the preprint in perpetuity. It is made available under aCC-BY-NC-ND 4.0 International license.

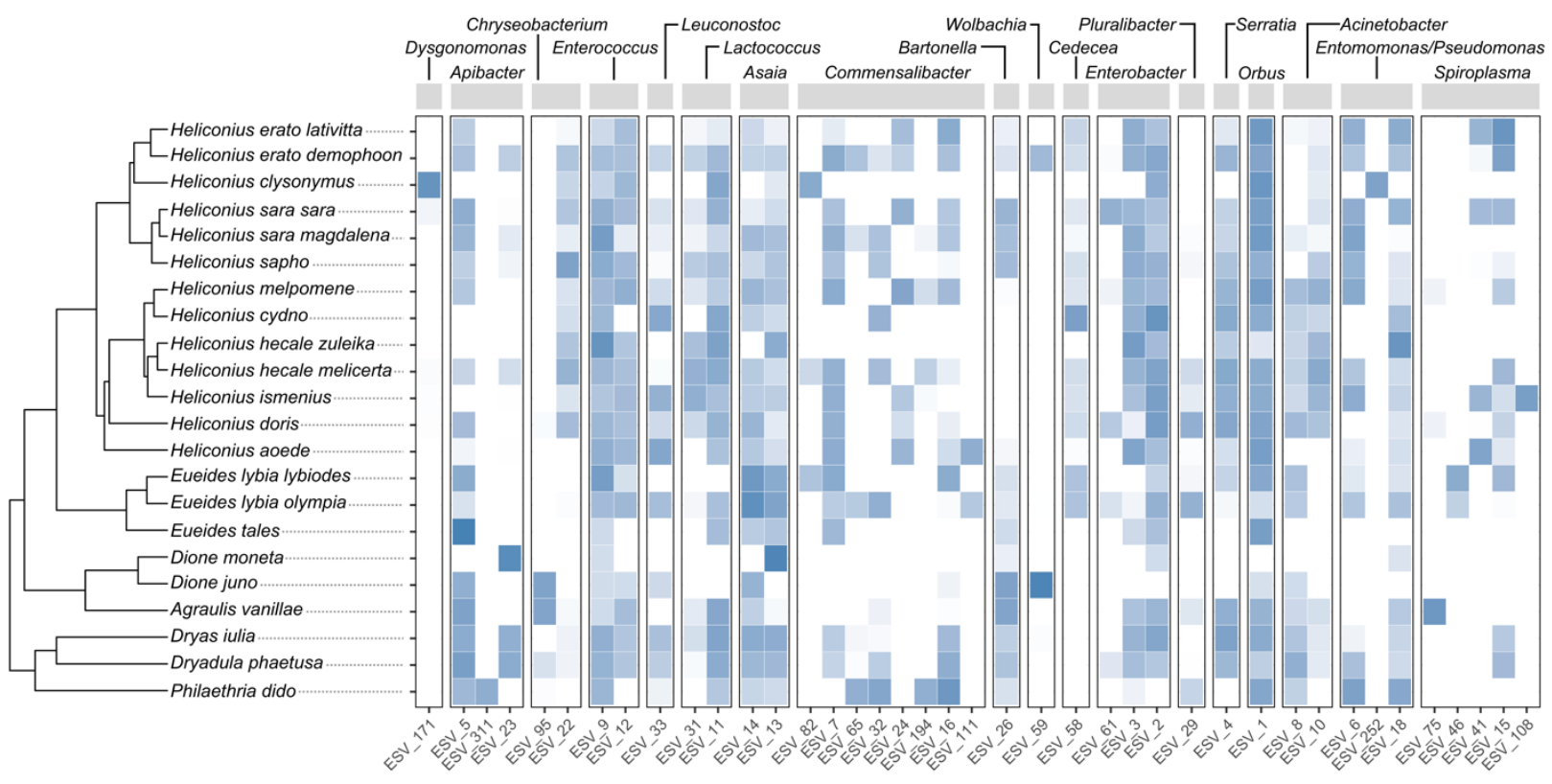

Figure 4. Most bacterial exact sequence variants (ESVs) are not host species- or host genus-specific. Shown are all ESVs in the dataset that had $\geq 5 \%$ mean relative abundance across conspecific individuals for one or more host species. For each ESV, the species-level mean relative abundance in whole-body samples, after $\log$ transformation, is indicated by the color of the cells (white $=$ not detected in that species). Note that bacterial genera (labeled at the top) contained varying numbers of ESVs that met the aforementioned prevalence threshold. 
Figure 5. Maximum likelihood phylogenetic reconstruction of the bacterial family Orbaceae (Gammaproteobacteria: Pasteurellales). Some host taxonomic structure is apparent at the order level (i.e. Lepidoptera, beetles, bees, flies) but not within butterflies. 16S rRNA gene sequences from Agraulis, Dryadula, Dryas, and Heliconius were assembled from short metagenomic reads. Other 16S rRNA gene sequences are from GenBank. Branch support values are shown next to nodes. Haemophilus influenzae (Pasteurellaceae) was used as the outgroup. 


\section{Supplementary Figures}

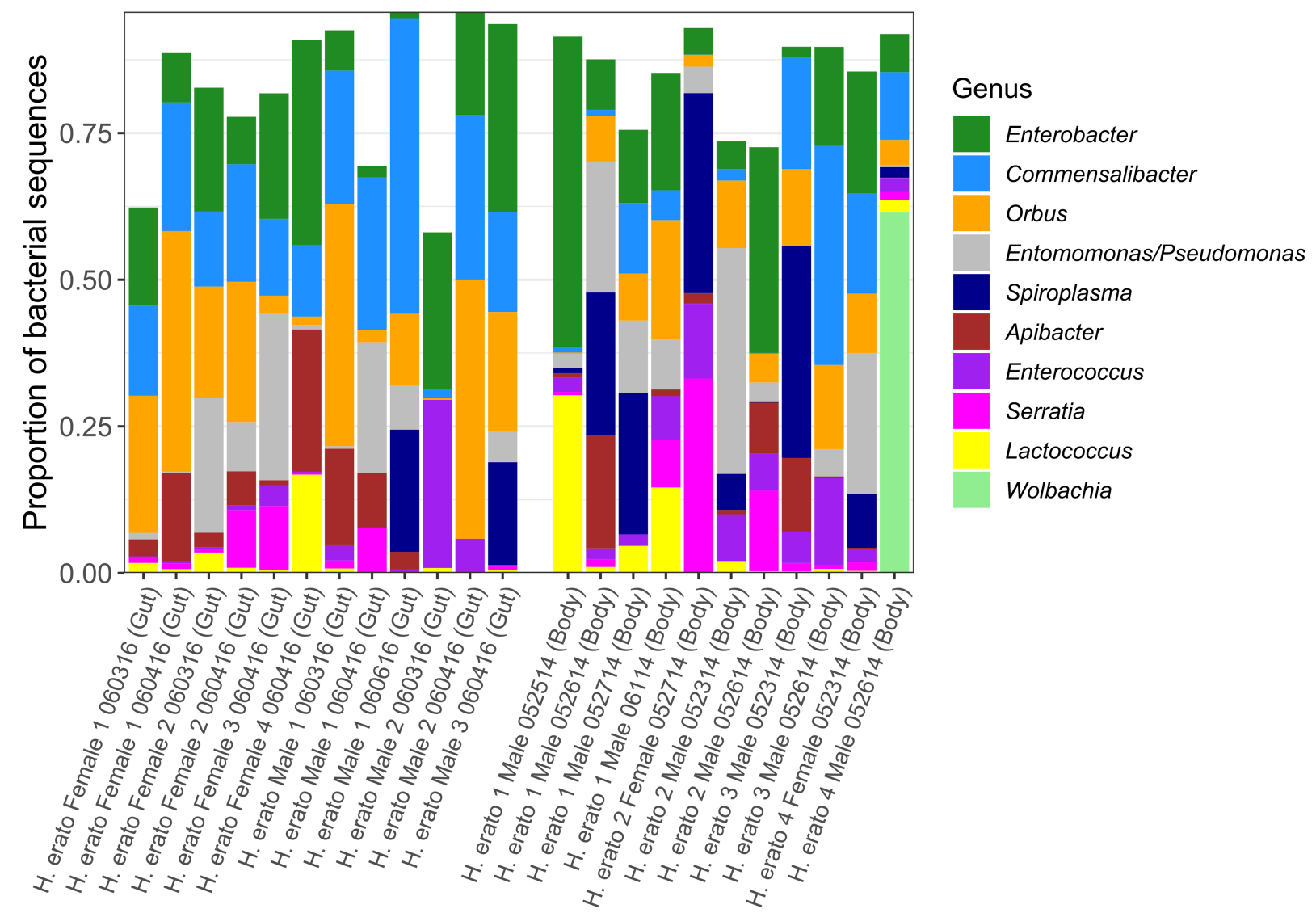

Figure S1. Whole-body bacterial communities are consistent among individuals and predominantly represent gut-associated taxa. Shown are the relative abundances of the top 10 bacterial genera (ranked by mean abundance) across $\mathrm{H}$. erato demophoon collected from Gamboa, Panama. Remaining white space represents sequences belonging to other genera; these made up a median $16 \%$ of sequence libraries across individuals. Samples on the left are isolated guts from individuals collected in 2016, while samples on the right are whole-body homogenates from individuals collected in 2014. Note that Enterobacter here includes sequences originally assigned as Klebsiella and some other closely related Enterobacteriaceae genera (see Methods). 

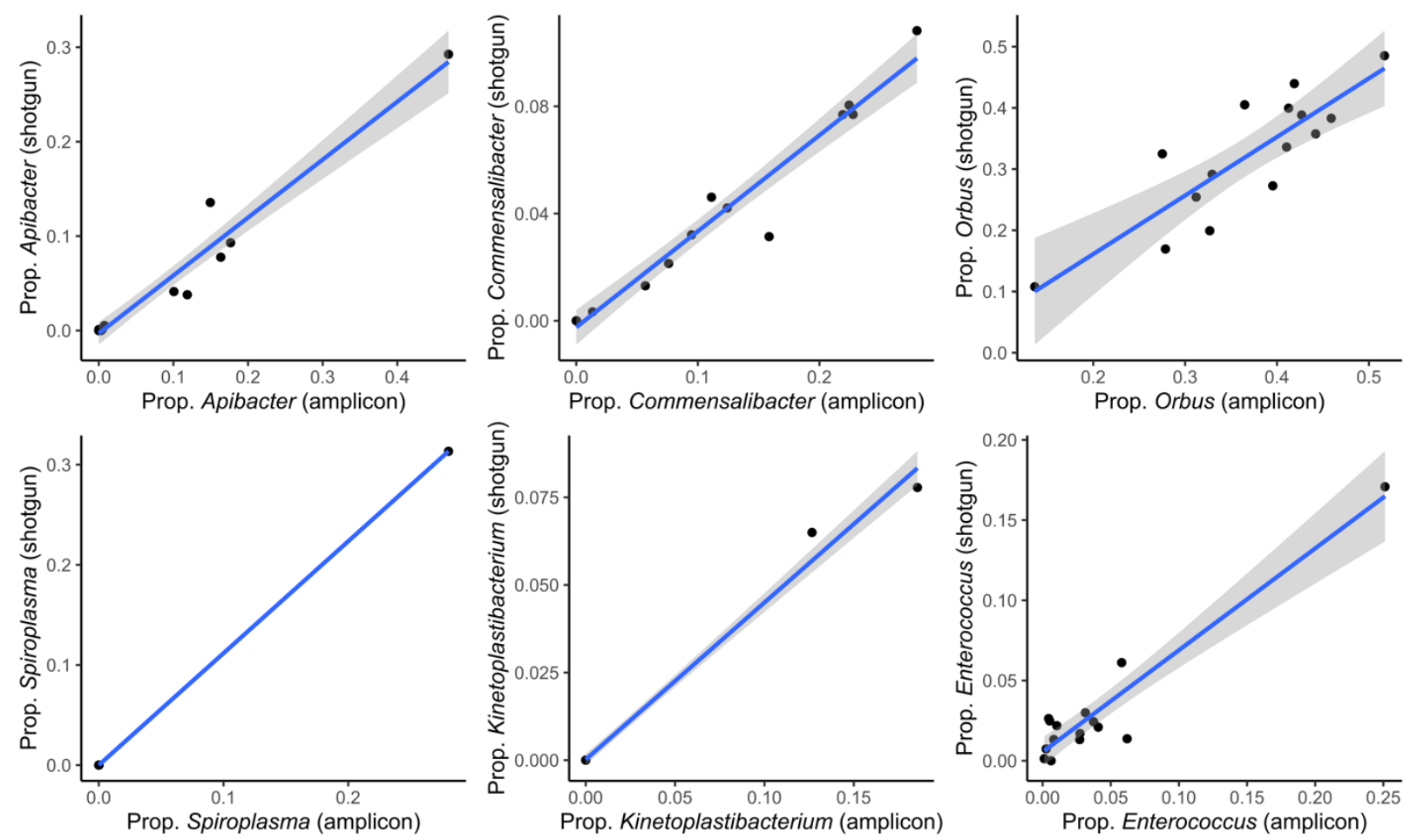

Figure S2. Bacterial genus-level relative abundances are highly correlated between the amplicon sequence libraries and metagenomes. Shown are relative abundances of six of the most abundant genera across the 15 butterfly gut samples for which we obtained both amplicon and shotgun metagenomic sequence data. 


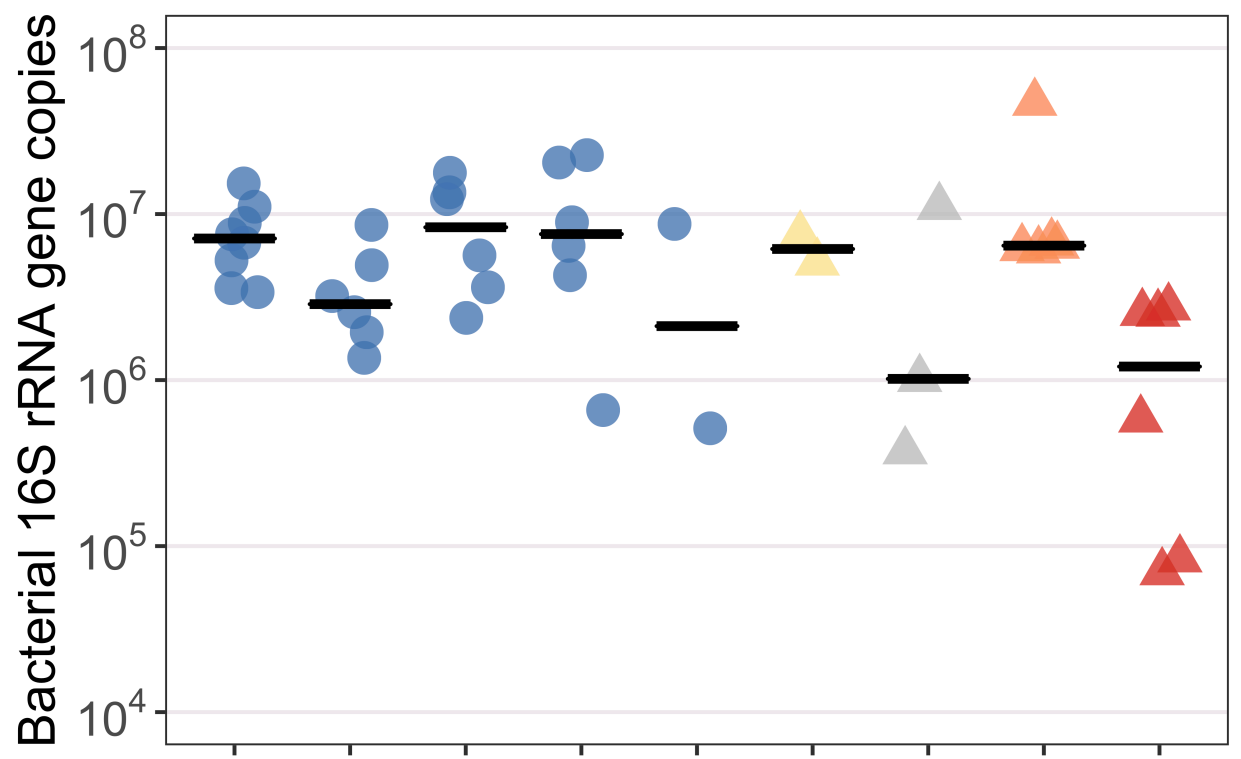

Host genus

Heliconius

Eueides

Agraulis

Dryas

Dryadula

Pollen-feeding

$\triangle$ No
Yes

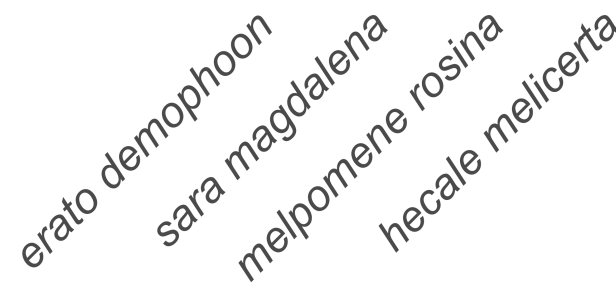<smiles></smiles><smiles>C#CCOCC#N</smiles>

\section{Host species}

Figure S3. Absolute abundances of bacteria in the head and thorax of adult heliconiine butterflies, derived from qPCR. Shown are the number of bacterial 16S rRNA gene copies per subsample (approx. $50 \mathrm{mg}$ ) of homogenized, combined head and thorax tissue. These individuals were collected from Gamboa, Panama in $2016(\mathrm{~N}=28$ Heliconius, 16 other Heliconiini). 


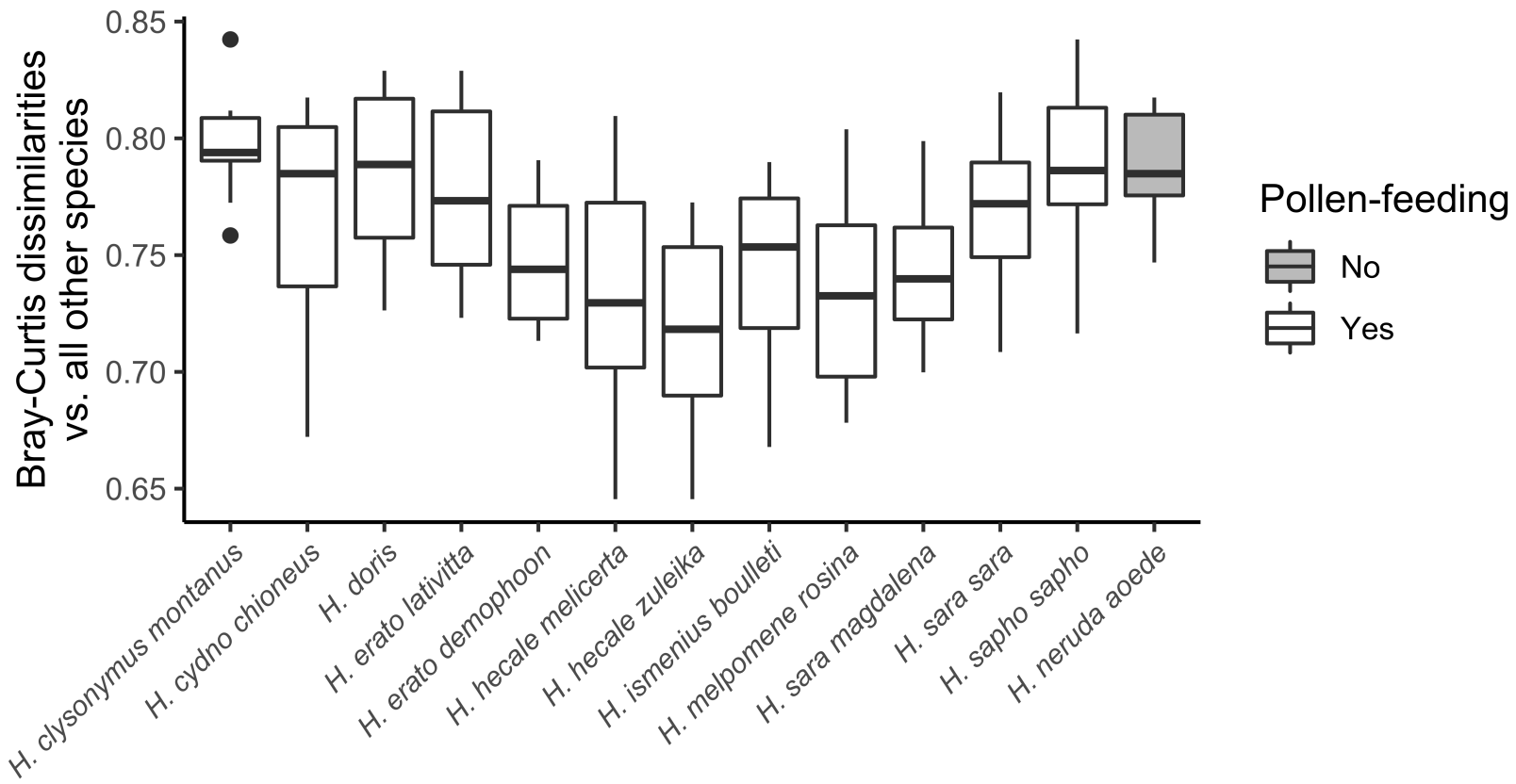

Figure S4. The non-pollen-feeding species Heliconius aoede is not uniquely distinct from pollen-feeding Heliconius species. Whole-body microbiomes from each focal species (x axis) are compared to all other species using Bray-Curtis dissimilarities. For a given comparison of two species' microbiomes, intraspecific replication was handled by averaging dissimilarities among all pairs of individuals. 


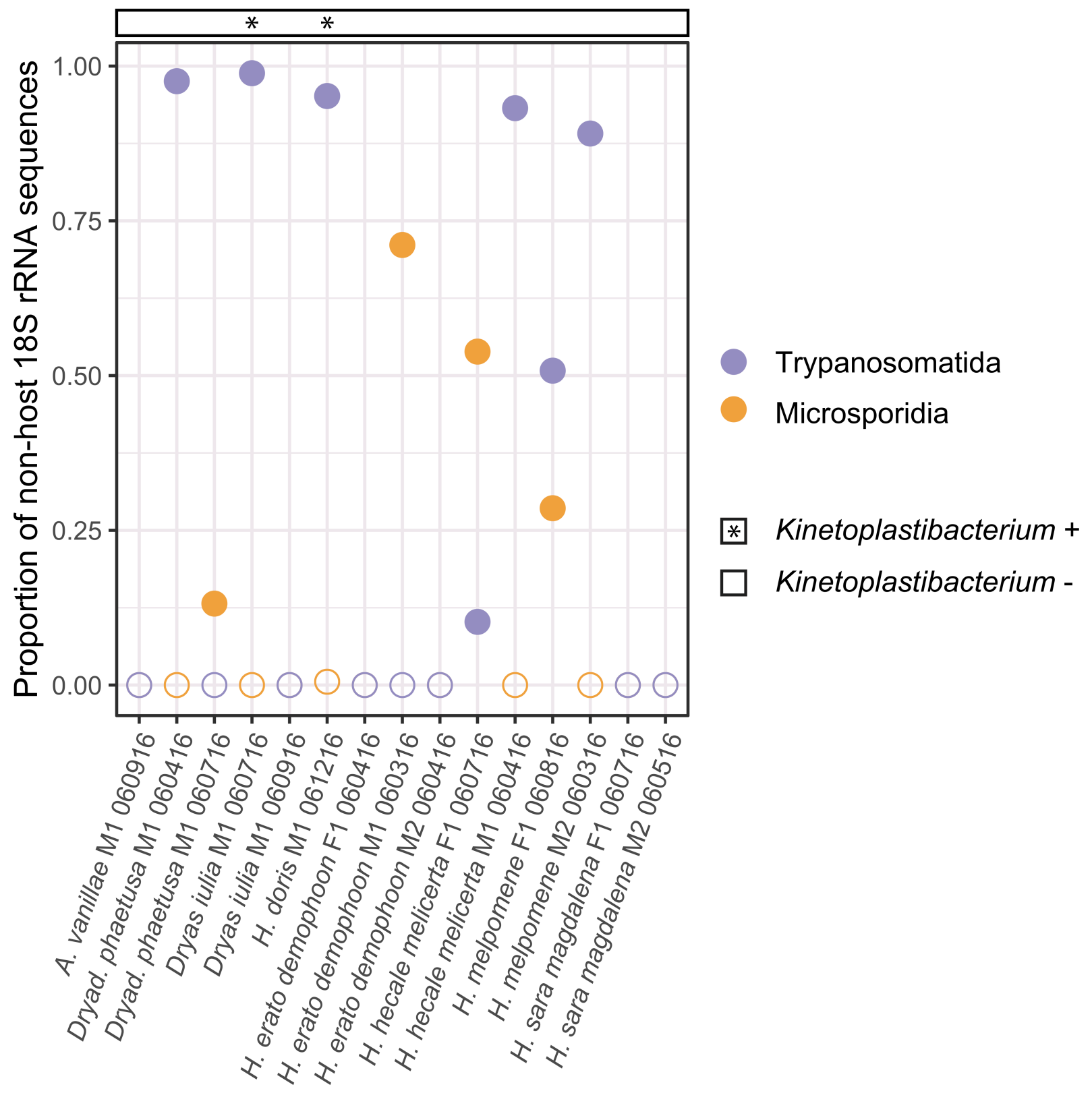

Figure S5. Microsporidia and Trypanosomatidae (related to Crithidia, Leishmania, and Trypanosoma) are prevalent in the 15 butterfly individuals with sequenced gut metagenomes. Points show the proportion of each microeukaryotic taxon out of all non-host $18 \mathrm{~S}$ rRNA sequences identified by phyloFlash. Circles are filled if the proportion of microsporidia or trypanosomatids exceeded 0.01. Asterisks indicate samples in which Kinetoplastibacterium, a bacterial endosymbiont of trypanosomatids, was detected in metagenomes. Other microeukaryotes not shown here comprise a variety of very low-abundance taxa (i.e. $\leq 30$ total reads per metagenome) classified mainly as coccidia, ascomycete fungi, acanthamoeba, and algae. 
bioRxiv preprint doi: https://doi.org/10.1101/820829; this version posted August 14, 2020. The copyright holder for this preprint (which was not certified by peer review) is the author/funder, who has granted bioRxiv a license to display the preprint in perpetuity. It is made available under aCC-BY-NC-ND 4.0 International license.

\section{Supplementary Table}

\begin{tabular}{|c|c|c|c|c|c|c|c|c|}
\hline Host taxon & Phylum & Class & Order & Family & Genus & $\begin{array}{l}\text { Mean prop. gut } \\
+/- \text { SEM }\end{array}$ & $\begin{array}{c}\text { Mean prop. head/thorax } \\
+/ \text { - SEM }\end{array}$ & p value \\
\hline \multirow[t]{15}{*}{ Heliconius } & Bacteroidetes & Bacteroidia & Flavobacteriales & Weeksellaceae & Apibacter & $0.036+/-0.009$ & $0.002+/-0.001$ & $<0.001$ \\
\hline & & & & & Chishuiella & $0.007+/-0.003$ & $0.095+/-0.027$ & 0.001 \\
\hline & & & & & Chryseobacterium & $0.011+/-0.004$ & $0.096+/-0.024$ & 0.001 \\
\hline & Firmicutes & Bacilli & Lactobacillales & Enterococcaceae & Enterococcus & $0.031+/-0.008$ & $0.021+/-0.006$ & 0.117 \\
\hline & & & & Streptococcaceae & Lactococcus & $0.02+/-0.006$ & $0.033+/-0.009$ & 0.793 \\
\hline & Proteobacteria & Alphaproteobacteria & Acetobacterales & Acetobacteraceae & Asaia & $0.024+/-0.007$ & $0.004+1-0.002$ & 0.001 \\
\hline & & & & & Commensalibacter & $0.12+/-0.02$ & $0.007+/-0.003$ & $<0.001$ \\
\hline & & & & & Swaminathania & $0.013+/-0.01$ & $0.016+/-0.014$ & 0.745 \\
\hline & & Gammaproteobacteria & Betaproteobacteriales & Burkholderiaceae & Variovorax & $0.01+/-0.004$ & $0.021+/-0.006$ & 0.017 \\
\hline & & & Enterobacteriales & Enterobacteriaceae & Enterobacter & $0.233+/-0.034$ & $0.031+/-0.007$ & $<0.001$ \\
\hline & & & & & Serratia & $0.037+/-0.01$ & $0.027+/-0.017$ & 0.013 \\
\hline & & & Orbales & Orbaceae & Orbus & $0.163+/-0.024$ & $0.021+/-0.01$ & $<0.001$ \\
\hline & & & Pseudomonadales & Moraxellaceae & Acinetobacter & $0.023+/-0.007$ & $0.293+/-0.041$ & $<0.001$ \\
\hline & & & & Pseudomonadaceae & Entomomonas/Pseudomonas & $0.086+/-0.017$ & $0.024+/-0.01$ & 0.291 \\
\hline & Tenericutes & Mollicutes & Entomoplasmatales & Spiroplasmataceae & Spiroplasma & $0.012+/-0.007$ & $0.003+/-0.002$ & 0.927 \\
\hline \multirow{15}{*}{ Other Heliconiini } & Bacteroidetes & Bacteroidia & Flavobacteriales & Weeksellaceae & Apibacter & $0.139+/-0.045$ & $0.008+/-0.004$ & 0.083 \\
\hline & & & & & Chishuiella & $0.009+/-0.003$ & $0.138+/-0.029$ & 0.016 \\
\hline & & & & & Chryseobacterium & $0+1-0$ & $0.015+/-0.012$ & 0.808 \\
\hline & Firmicutes & Bacilli & Lactobacillales & Enterococcaceae & Enterococcus & $0.056+/-0.027$ & $0.031+/-0.011$ & 0.843 \\
\hline & & & & Streptococcaceae & Lactococcus & $0.036+/-0.026$ & $0.012+/-0.011$ & 0.098 \\
\hline & Proteobacteria & Alphaproteobacteria & Acetobacterales & Acetobacteraceae & Asaia & $0.153+/-0.039$ & $0.004+/-0.001$ & 0.01 \\
\hline & & & & & Commensalibacter & $0.031+/-0.015$ & $0.002+/-0.002$ & 0.106 \\
\hline & & & & & Swaminathania & $0.007+/-0.004$ & $0+1-0$ & 0.226 \\
\hline & & Gammaproteobacteria & Betaproteobacteriales & Burkholderi & Variovorax & $0.005+/-0.002$ & $0.016+/-0.004$ & 0.292 \\
\hline & & & Enterobacteriales & Enterobacteriaceae & Enterobacter & $0.156+/-0.042$ & $0.014+/-0.006$ & 0.01 \\
\hline & & & & & Serratia & $0.02+/-0.012$ & $0.001+1-0$ & 0.075 \\
\hline & & & Orbale & Orba & Orbus & $0.139+/-0.043$ & $0.007+1-0.004$ & 0.042 \\
\hline & & & Pseudomonadales & Moraxellaceae & Acinetobacter & $0.046+/-0.018$ & $0.45+/-0.054$ & $<0.001$ \\
\hline & & & & Pseudomonadaceae & Entomomonas/Pseud & $0.02+/-0.017$ & $0.011+/-0.004$ & 0.393 \\
\hline & Tenericutes & Mollicutes & Entomoplasmatales & Spiroplasmataceae & Spiroplasma & $0.068+1-0.038$ & $0.052+/-0.052$ & 0.843 \\
\hline
\end{tabular}

Table S1. Relative abundances and within-body distribution of the top 15 bacterial genera (ranked by mean abundance) across butterflies collected from Gamboa, Panama in 2016. These individuals were dissected to compare microbiomes between isolated gut tissue and the combined head and thorax. Abundances are shown for Heliconius (top) and species belonging to other heliconiine genera (bottom) separately. P values are from a nonparametric statistical test of proportions in guts versus head/thorax samples, after FDR correction (see Methods). 